\title{
Reversion of left ventricle remodeling in spontaneously hypertensive rats by valsartan is associated with the inhibition of caspase- $3,-8$ and -9 activities
}

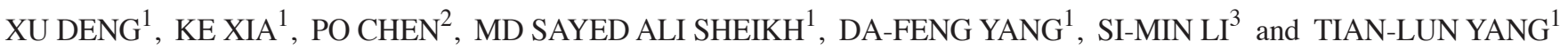 \\ ${ }^{1}$ Department of Cardiology, Xiangya Hospital; ${ }^{2}$ Hunan Provincial Tumor Hospital; ${ }^{3}$ Xiangya Stomatological Hospital, \\ Central South University, Changsha, Hunan 410008, P.R. China
}

Received January 30, 2015; Accepted April 8, 2015

DOI: $10.3892 /$ br. 2015.458

\begin{abstract}
The development of hypertension is closely associated with cardiac hypertrophy and apoptosis, and caspase-3, -8 and -9 are key enzymes of apoptosis. The aim of the present study was to evaluate the effects of valsartan on left ventricle hypertrophy and myocardial apoptosis in spontaneously hypertensive rats (SHRs) and to explore the mechanisms for valsartan against apoptosis. A total of 15 SHRs (16 weeks old) were randomly divided into two groups. The SHRs in the valsartan $(n=8)$ and SHR groups $(n=7)$ were fed with valsartan and distilled water for 8 weeks, respectively. Wistar-Kyoto rats $(n=8)$ were the control group. At the end of the experiments, blood pressure, parameters regarding hypertrophy, apoptosis and activities of caspase-3, -8 and -9 were measured. The results showed that valsartan significantly reduced systolic blood pressure and left ventricular hypertrophy, improved left ventricular remodeling, attenuated the myocardial damage and apoptosis, and decreased the activities of caspase-3, -8 and -9 in SHRs. In conclusion, valsartan is able to reverse hypertension-induced left ventricle remodeling, which is associated with, at least in part, its inhibitory effect on myocardial apoptosis in the death receptor-mediated extrinsic, as well as the mitochondrial-mediated intrinsic pathways.
\end{abstract}

\section{Introduction}

Hypertension is an important risk factor for cardiovascular disease events (1) and left ventricular hypertrophy exists in approximately one-third of hypertensive patients (2).

Correspondence to: Professor Tian-Lun Yang, Department of Cardiology, Xiangya Hospital, Central South University, 87 Xiangya Road, Changsha, Hunan 410008, P.R. China

E-mail: tianluny@163.com

Key words: caspase-3, -8 and -9, hypertension, cardiac hypertrophy, valsartan, apoptosis
Left ventricular hypertrophy resulting from hypertension is recognized as an independent risk factor for cardiovascular complications (3). Cellular proliferations are closely associated with the progression of hypertension, myocardial hypertrophy and apoptosis. In the early stage of hypertension, there are more myocardial proliferations than apoptosis. With the development of hypertension, apoptosis becomes the dominant position (4).

Since the caspase family was first identified in 1992, 15 members of this family in mammalian cells have been found. Certain members of the caspase family play a vital role in cytokine maturation and inflammation, while some participate in apoptosis (5). For those apoptosis-related caspases, they were divided into two groups; executioner and initiator caspases (6). Initiator caspases possess a death effectors domain or the caspase-recruitment domain, which are responsible for receiving death-inducing signaling complexes. Executioner caspases carry out the program of apoptosis, causing changes in cell morphology (7). In the caspase-dependent apoptotic pathway, caspase- 3 is the core of the executioner caspases, while caspase- 8 and -9 are the representatives of the initiator caspases. In general, there are two pathways that can be activated through the caspase. One is the death receptor-mediated pathway (such as the caspase-8-dependent pathway) and another is the mitochondrion-mediated pathway (such as the caspase-9-dependent pathway) (8).

The aims for hypertension treatment are not only to lower blood pressure, but also to reverse the left ventricular hypertrophy and to delay the occurrence and development of heart failure. Valsartan, an angiotensin II antagonist, is widely used in the control of hypertension. It has been shown that valsartan can inhibit myocardial apoptosis (9) and improve left ventricular remodeling (10). However, it is not known that valsartan inhibits myocardial apoptosis through the death receptor-mediated extrinsic pathway or mitochondrial-mediated intrinsic pathway.

The main aims of the present study were to observe the effect of valsartan on left ventricular hypertrophy, systolic blood pressure (SBP) and myocardial apoptosis in spontaneously hypertensive rats (SHRs), as well as to clarify the involved caspase-mediated apoptosis signal pathways. 


\section{Materials and methods}

Animal care. Sixteen-week-old male SHRs and Wistar-Kyoto (WKY) rats (280-320 g) provided by the Animal Center of Central South University (Changsha, China) were housed at a constant temperature $\left(22^{\circ} \mathrm{C}\right)$ with a 12 -h light/dark cycle. Animal care was in compliance with the Guidelines for the Care and Use of Laboratory Animals.

Animal treatment. SHRs were randomly divided into two groups: i) The valsartan group (SHR + valsartan, $n=8)$, rats were fed with valsartan (Novartis Pharma Ltd., Beijing, China) using a stomach tube (30 mg/kg/day intragastrically) for 8 weeks; and ii) the SHR group $(n=7)$, rats were fed with distilled water. In addition, $8 \mathrm{WKY}$ rats served as the control group, and they were also fed with distilled water. At the end of the experiments, the left ventricular myocardial tissues were collected. Some were embedded with paraffin and some were stored at $-80^{\circ} \mathrm{C}$ for further studies.

SBP and left ventricular mass index. The weight and caudal artery SBP of the rats were recorded at the end of the experiments. The left ventricles were isolated, washed with normal saline solution and weighed.

Hematoxylin and eosin $(H \& E)$ staining. The left ventricular tissues were fixed in $4 \%$ paraformaldehyde and embedded in paraffin. The slices underwent $H \& E$ staining according to protocol. Following H\&E staining, the results as images were captured by microscope to assess the morphological changes among the experimental groups.

Tunel assay. Three paraffin sections randomly picked up from the left ventricular tissue randomly were analyzed by a terminal deoxynucleotidyl transferase-mediated dUTP nick end labeling (TUNEL) assay according to the manufacturer's instructions. The TUNEL index (\%) was calculated as the ratio of the number of TUNEL-positive cells divided by the total number of cells. At least 3 representative fields were evaluated for each experimental group, from which an average value was calculated.

Activities of caspase-3, -8 and -9. Frozen myocardial tissues were rinsed in cold phosphate-buffered saline and crushed in liquid nitrogen. Tissue samples were homogenized in lysis buffer and protein concentrations in the supernatant were determined by the Bradford assay. Supernatant $(50 \mu \mathrm{l})$ together with $5 \mu \mathrm{l}$ of caspase- $3,-8$ or -9 substrates was incubated at $37^{\circ} \mathrm{C}$ for $4 \mathrm{~h}$ in darkness. The optical density (OD) was determined at $405 \mathrm{~nm}$ by a microplate reader and the activity of caspase was presented by calculating the ratio of OD (sample)/OD (negative).

Statistical analysis. All the data are presented as mean \pm standard error. Comparisons between the valsartan and SHR groups, and SHR and control groups were assessed by independent-samples t-test. Comparisons between the valsartan and SHR groups were assessed by paired-samples t-test. Pearson correlation coefficients were calculated when indicated. $\mathrm{P}<0.05$ was considered to indicate a statistically significant difference. For statistical analysis, the SPSS version 19.0 software (IBM, Corp., Armonk, NY, USA) was used.
Table I. Effects of valsartan on SBP and LV mass index.

\begin{tabular}{lclll}
\hline Group & No. & SBP, mmHg & BW, g & LV weight/BW, mg/g \\
\hline Control & 8 & $118.3 \pm 4.5$ & $368 \pm 19.5$ & $3.2 \pm 0.28$ \\
SHR & 7 & $192.4 \pm 6.1^{\mathrm{a}}$ & $342 \pm 22.7^{\mathrm{a}}$ & $4.6 \pm 0.31^{\mathrm{a}}$ \\
Valsartan & 8 & $161.0 \pm 5.7^{\mathrm{a}, \mathrm{b}}$ & $345 \pm 21.8^{\mathrm{a}}$ & $3.9 \pm 0.30^{\mathrm{a}, \mathrm{b}}$ \\
\hline
\end{tabular}

aSignificantly different $(\mathrm{P}<0.05)$ from control, ${ }^{\mathrm{b}} \mathrm{P}<0.05$ vs. SHR. Values are mean \pm standard error. Control, indicates Wistar-Kyoto rats; SHR, spontaneously hypertensive rats; SBP, systolic blood pressure; valsartan, SHRs were fed with valsartan; LV, left ventricular; BW, body weight.

\section{Results}

SBP and left ventricular mass index. There was no significant difference in body weight between the SHR and valsartan groups. The SBP was significantly elevated in the SHR group compared with that in the control group, which was evidently attenuated by valsartan. The left ventricular remodeling was evaluated by comparing the left ventricular mass index, which was the ratio of the left ventricular weight to body weight. The index was significantly increased in the SHR group compared with that in the control group, which was clearly reversed in the presence of valsartan (Table I).

Morphological changes of myocardial tissues. In the control group, the left ventricular myocardial fibers were arranged in neat rows and intact borders, with little congestion, edema and inflammatory cell infiltration. By contrast, myocardial tissues in the SHR group showed disorganized myocardial cells, deep-dye nucleus, swelling myocardial interstitium and capillary hyperemia. These phenomena were significantly improved following valsartan treatment (Fig. 1).

Cellular apoptosis in myocardial tissues. As shown in Fig. 2, the black arrows indicate the non-apoptotic cells while the white arrows indicate the apoptotic cells. Compared to the control group, the apoptotic index in the SHR group was significantly increased. This increase was evidently attenuated in the presence of valsartan (Fig. 3).

Activities of caspase-3, -8 and -9 . The activities of caspase-3, - 8 and -9 were determined by calculating the ratio of OD (sample)/OD (negative). As shown in Table II, the activities of caspase-3, -8 and -9 in the SHR group were dramatically elevated compared to that in the control group, which were markedly reduced by valsartan treatment.

\section{Discussion}

Hypertension is an important cause and risk factor for various cardiovascular diseases (11). In recent years, numerous studies demonstrated that high blood pressure induced myocardial apoptosis $(9,12,13)$. The caspase is involved in apoptosis, and therefore it was used in the present study as a marker of apoptosis. Previous studies showed that valsartan, an angiotensin II antagonist, could exert an effect on apoptosis and left 

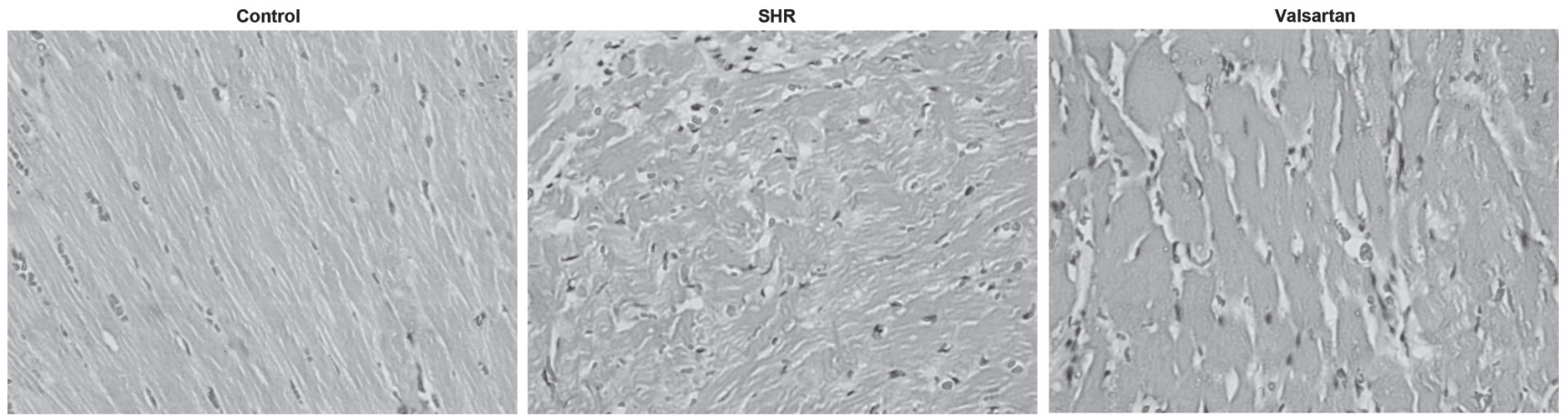

Figure 1. Hematoxylin and eosin staining of myocardial tissue under inverted phase contrast microscope. Images were captured at magnification, $\mathrm{x} 400$.
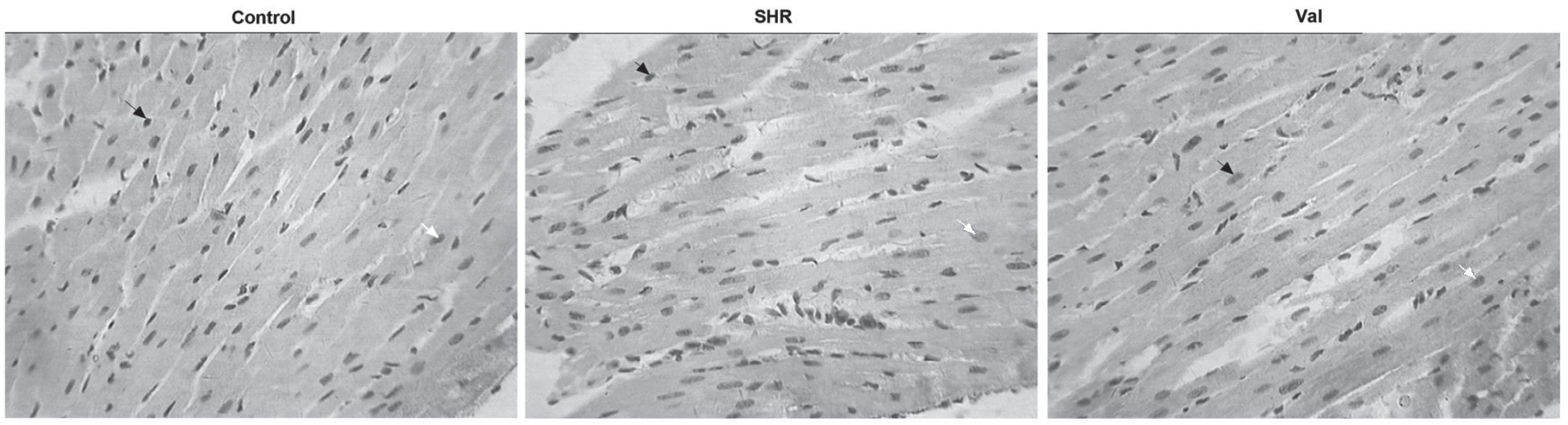

Figure 2. Myocardial tissue with terminal deoxynucleotidyl transferase-mediated dUTP nick end labeling assay. Images were captured at magnification, $\mathrm{x} 400$.

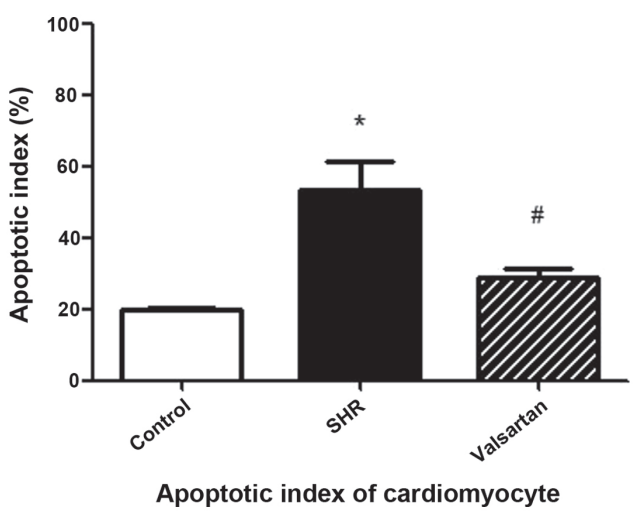

Figure 3. Cardiomyocyte apoptotic index detected by the terminal deoxynucleotidyl transferase-mediated dUTP nick end labeling method. Control, indicates Wistar-Kyoto rats; SHR, spontaneously hypertensive rats; valsartan, SHRs were fed with valsartan. "Significantly different $(\mathrm{P}<0.05)$ from control, ${ }^{*} \mathrm{P}<0.05$ vs. SHR.

ventricular remodeling $(10,14,15)$. However, the underlying mechanisms remain unclear.

The caspase family is involved in two major functions including inflammation and apoptosis. However, recent studies focus more on their role in apoptosis. Caspases can be classified into initiator and executioner caspases (16). Caspases-8 and -9 are typical initiator caspases, with an extremely long and functionally pro-domain, to initiate and regulate apoptosis. However, the pro-domain of executioner caspases is extremely short. They act downstream in the common pathway, amplifying signals from intrinsic or extrinsic pathways, carrying
Table II. Activity of caspase- $3,-8$ and -9 among the different groups.

\begin{tabular}{lcccc}
\hline Group & No. & Caspase-3 & Caspase- 8 & Caspase- 9 \\
\hline Control & 8 & $5.10 \pm 0.68$ & $5.43 \pm 0.84$ & $5.23 \pm 0.41$ \\
SHR & 7 & $11.21 \pm 1.05^{\mathrm{a}}$ & $10.92 \pm 0.74^{\mathrm{a}}$ & $11.50 \pm 0.80^{\mathrm{a}}$ \\
Valsartan & 8 & $7.86 \pm 1.18^{\mathrm{a}, \mathrm{b}}$ & $7.63 \pm 0.66^{\mathrm{a}, \mathrm{b}}$ & $7.84 \pm 0.87^{\mathrm{a}, \mathrm{b}}$ \\
\hline
\end{tabular}

${ }^{a}$ Significantly different $(\mathrm{P}<0.05)$ from control, ${ }^{\mathrm{b}} \mathrm{P}<0.05$ vs. SHR. Values are mean \pm standard error. Control, indicates Wistar-Kyoto rats; SHR, spontaneously hypertensive rats; valsartan, SHRs were fed with valsartan.

out the biochemical changes in apoptosis (17). Morphological changes of apoptosis appeared several hours previously, followed by the activation of downstream caspase-3, -6 and -7 , the cell death would be inevitable (18). Caspase-3, -6 and -7 , typical executioner caspases, must rely on the activation of initiator caspase, subsequent to performing their activities (19). There are two pathways in caspase-dependent apoptosis. One is the death signal-induced, death receptor-mediated pathway (such as the caspase-8-dependent pathway). Another is the stress-induced, mitochondrion-mediated pathway (such as the caspase-9-dependent pathway). In the two pathways, the downstream caspases, such as caspase-3, always play a central role in apoptosis (20). In the present study, the activity of caspase-3 in the left ventricle from SHR was significantly increased compared with the control rats, which was reversed 
by valsartan. The results indicated that hypertension-induced apoptosis was associated with caspases and valsartan can inhibit the caspase-dependent apoptosis pathway.

The activation of the extrinsic death receptor pathway is mediated by caspase- 8 (21) and the activation of the intrinsic mitochondrial pathway is mediated by caspase- $9(16,22)$. The present results showed that the activities of caspase- 8 and -9 in the left ventricle from the hypertensive rats were evidently elevated compared with the control rats. Valsartan treatment significantly reversed the increase of caspase- 8 and -9 activities. Together with the abovementioned results, the results suggest that hypertension-induced myocardial cell apoptosis is associated with the extrinsic death receptor pathway and intrinsic mitochondrial pathway.

In conclusion, valsartan is able to inhibit hypertension-induced left ventricular remodeling, which is associated, at least in part, to its inhibitory effect against myocardial apoptosis in the death receptor-mediated extrinsic, as well as the mitochondrial-mediated intrinsic pathways.

\section{Acknowledgements}

The present study was supported by the Chinese Medical Association (grant no. 08010008).

\section{References}

1. Franklin SS, Gokhale SS, Chow VH, Larson MG, Levy D, Vasan RS, Mitchell GF and Wong ND: Does low diastolic blood pressure contribute to the risk of recurrent hypertensive cardiovascular disease events? The Framingham Heart Study. Hypertension 65: 299-305, 2015.

2. Giles TD, Berk BC, Black HR, Cohn JN, Kostis JB, Izzo JL Jr and Weber MA: Expanding the definition and classification of hypertension. J Clin Hypertens (Greenwich) 7: 505-512, 2005.

3. Mathew J, Sleight P, Lonn E, Johnstone D, Pogue J, Yi Q, Bosch J, Sussex B, Probstfield J and Yusuf S; Heart Outcomes Prevention Evaluation (HOPE) Investigators: Reduction of cardiovascular risk by regression of electrocardiographic markers of left ventricular hypertrophy by the angiotensin-converting enzyme inhibitor ramipril. Circulation 104: 1615-1621, 2001.

4. Jiao Y and Hong X: Effect of heart remodeling and cardiac myocyte apoptosis in juvenile spontaneously hypertensive rats under the intervention of valsartan, enalapril and propranolol. Jiangsu Med J 29: 255-257, 2003.

5. Yamamoto-Tanaka M and Hibino T: Caspase-14 protocols. Methods Mol Biol 1133: 89-100, 2014.

6. McStay GP and Green DR: Detection of caspase activity using antibody-based techniques. Cold Spring Harb Protoc 2014: 783-788, 2014
7. Marani M, Tenev T, Hancock D, Downward J and Lemoine NR: Identification of novel isoforms of the $\mathrm{BH} 3$ domain protein Bim which directly activate Bax to trigger apoptosis. Mol Cell Biol 22: 3577-3589, 2002.

8. Fan TJ, Han LH, Cong RS and Liang J: Caspase family proteases and apoptosis. Acta Biochim Biophys Sin (Shanghai) 37: 719-727, 2005.

9. Li W, Sun N, Liu W, Chen Y and Yu Y: Influence of Valsartan on myocardial apoptosis in spontaneously hypertensive rats. Chin Med J (Engl) 115: 364-366, 2002.

10. Zhi-Bin H, Chang F, Mao-Huan L, Gui-Yi Y, Shu-Xian Z and Wei W: Valsartan improves the electrophysiological characteristics of left ventricular hypertrophic myocardium in spontaneously hypertensive rats. Hypertens Res 37: 824-829, 2014.

11. Gaziano TA: Reducing the growing burden of cardiovascular disease in the developing world. Health Aff (Millwood) 26: 13-24, 2007.

12. Correia-Pinto J, Henriques-Coelho T, Roncon-Albuquerque R Jr, Lourenço AP, Melo-Rocha G, Vasques-Nóvoa F, Gillebert TC and Leite-Moreira AF: Time course and mechanisms of left ventricular systolic and diastolic dysfunction in monocrotaline-induced pulmonary hypertension. Basic Res Cardiol 104: 535-545, 2009.

13. Kumar V, Bhandari U, Tripathi CD and Khanna G: Evaluation of antiobesity and cardioprotective effect of Gymnema sylvestre extract in murine model. Indian J Pharmacol 44: 607-613, 2012.

14. Der Sarkissian S, Tea BS, Touyz RM, deBlois D and Hale TM: Role of angiotensin II type 2 receptor during regression of cardiac hypertrophy in spontaneously hypertensive rats. J Am Soc Hypertens 7: 118-127, 2013.

15. Akashiba A, Ono H, Ono Y, Ishimitsu $\mathrm{T}$ and Matsuoka $\mathrm{H}$ : Valsartan improves L-NAME-exacerbated cardiac fibrosis with TGF- $\beta$ inhibition and apoptosis induction in spontaneously hypertensive rats. J Cardiol 52: 239-246, 2008.

16. Liu X, Kim CN, Yang J, Jemmerson R and Wang X: Induction of apoptotic program in cell-free extracts: Requirement for dATP and cytochrome c. Cell 86: 147-157, 1996.

17. Singh SS and Kang PM: Mechanisms and inhibitors of apoptosis in cardiovascular diseases. Curr Pharm Des 17: 1783-1793, 2011

18. Brunet CL, Gunby RH, Benson RS, Hickman JA, Watson AJ and Brady G: Commitment to cell death measured by loss of clonogenicity is separable from the appearance of apoptotic markers. Cell Death Differ 5: 107-115, 1998

19. Kim NH and Kang PM: Apoptosis in cardiovascular diseases: Mechanism and clinical implications. Korean Circ J 40: 299-305, 2010.

20. Haunstetter A and Izumo S: Apoptosis: Basic mechanisms and implications for cardiovascular disease. Circ Res 82: 1111-1129, 1998.

21. Chen G and Goeddel DV: TNF-R1 signaling: A beautiful pathway. Science 296: 1634-1635, 2002.

22. Li P, Nijhawan D, Budihardjo I, Srinivasula SM, Ahmad M, Alnemri ES and Wang X: Cytochrome $\mathrm{c}$ and dATP-dependent formation of Apaf-1/caspase-9 complex initiates an apoptotic protease cascade. Cell 91: 479-489, 1997. 